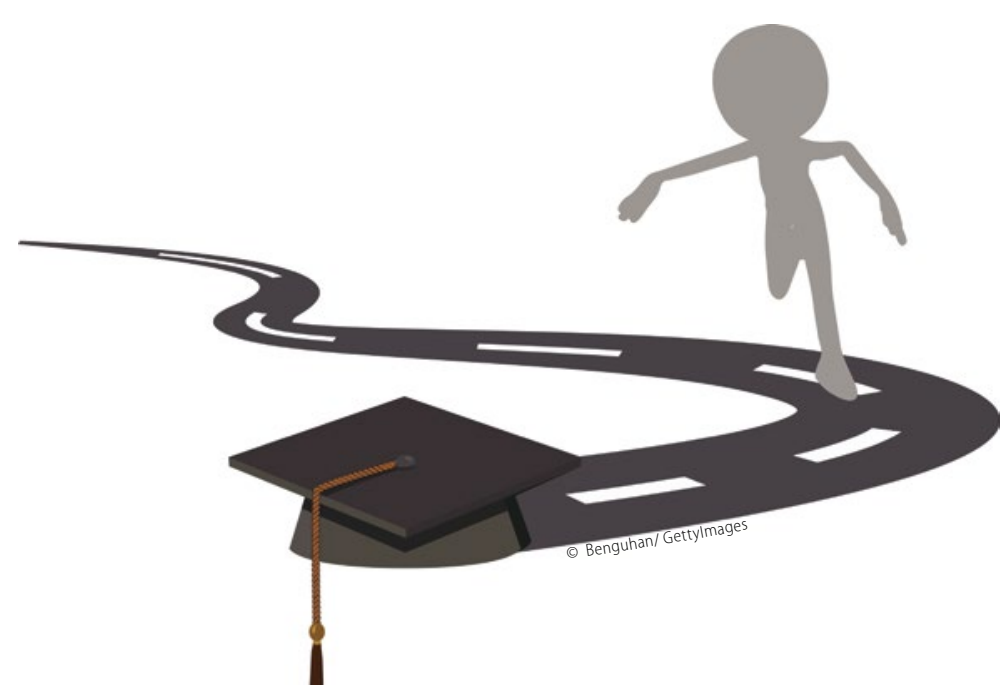

\title{
Schmalspur-Zahnmedizin ist keine Lösung
}

Liebe Kolleginnen und Kollegen,

der Wunsch des Staates nach weiteren Kostendämpfungen im Gesundheitswesen - natürlich bei gleichbleibender Qualität - setzt sich fort. Die mit Vorliebe dafür verwendeten Instrumente (nach Honorarkürzungen und Budgets) sind Qualitätsmanagement, Qualitätssicherung, Hygienerichtlinien und überbordende Bürokratie. Die Lösung soll nun in der Akademisierung der gesundheitlichen Hilfsberufe liegen. Aber wird das funktionieren?

Der Wissenschaftsrat hat in seinen „Empfehlungen zur hochschulischen Qualifikation für das Gesundheitswesen“ von 2012 deutliche Empfehlungen ausgesprochen. Er ist unter anderem der Meinung, dass die Angehörigen der Gesundheitsversorgungsberufe künftig an Hochschulen auszubilden sind (Bachelor). Auch hat er einige Empfehlungen zur strukturellen Weiterentwicklung der ärztlichen und zahnärztlichen Qualifizierung abgegeben. Da mit einer Modernisierung der zahnärztlichen Approbationsordnung in der nächsten Zeit nicht zu rechnen ist, besteht zunehmend die Gefahr, dass das Zahnmedizinstudium auf ein Bachelor- und Master-Studium umgestellt wird. Auch beim Wissenschaftsrat sind solche Tendenzen zu erkennen.

\section{Bachelor als Antwort auf erhöhten Bedarf}

Das Gesundheitswesen steht in den kommenden Jahren vor großen Herausforderungen. Als Folge des demographischen Wandels entsteht in der Zahnmedizin ein besonderer Versorgungsbedarf bei Patienten mit chronischen (Mehrfach-)Erkrankungen und/oder einer hohen Pflegebedürftigkeit. Dies bedeutet unter anderem einen erhöhten Bedarf im Bereich der Prophylaxe bis ins hohe Alter. Der Wissenschaftsrat sieht hier eine wachsende Bedeutung der Berufsgruppe der Dentalhygienikerinnen und Dentalhygieniker( DH). Da die DH bisher jedoch fast ausschließlich durch Fortbildungen der Landeszahnärztekammern ausgebildete zahnmedizinische Fachangestellte (ZFA) sind, fehlen ihnen eigene berufsrechtliche Grundlagen. Bereits 2005 hat der Wissenschaftsrat eine schrittweise Anhebung des Qualifizierungsniveaus empfohlen. Zudem wurde der Aufbau von Fachschulen an universitären Standorten der Zahnmedizin und die Entwicklung von weiterbildenden Studienangeboten an Hochschulen angeregt.

Die eigentliche Frage muss jedoch zunächst sein, ob man für diese Präventionsaufgaben wirklich akademisch ausgebildete DH braucht oder ob diese Aufgaben nicht auch von gut ausgebildeten zahnmedizinischen Prophylaxehelferinnen und -helfern (ZMP) ausgeführt werden können. Ist es wirklich sinnvoll,

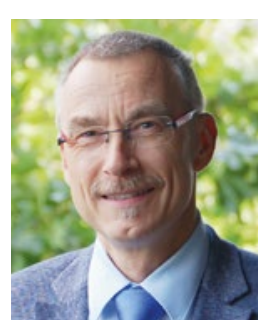

drs. Hub. van Rijt

Mitglied im FVDZ-Bundesvorstand

dafür selbstständig tätige DH, überspitzt gesagt: „Mini-Zahnärzte", auszubilden oder ist es besser, die Prophylaxe in Kombination mit den regelmäßigen Kontrolluntersuchungen des Zahnarztes durch ZMP durchführen zu lassen? In der Politik mag man der Meinung sein, dass es ausreicht, wenn in solchen Situationen „ein bisschen gereinigt und zur Not noch eine kleine Füllung gemacht wird“. Damit soll dann das Problem gelöst sein.

\section{Mehr Probleme als Lösungen}

Mitnichten, liebe Kolleginnen und Kollegen! Gerade Patienten mit chronischen (Mehrfach-)Erkrankungen und Pflegebedürftigkeit haben häufig komplizierte zahnmedizinische Probleme, die durch eine "Schmalspur-Zahnmedizin“ von Bachelor-DH (oder später vielleicht einem Bachelor-Zahnarzt, wer weiß?) nicht zu bewältigen sind. Zudem: Was passiert, wenn BachelorDH sich nicht (wie gewünscht) hauptsächlich um Pflegebedürftige kümmern, sondern Zahnreininungsinstitute als eine Art „Praxis für Schmalspur-Zahnmedizin“ aufmachen? Hier wähnt sich der Patient dann eventuell in der trügerischen Sicherheit, auf einen Zahnarztbesuch verzichten zu können - mit möglicherweise katastrophalen Folgen für seine Mundgesundheit.

Die Akademisierung der Heilhilfsberufe löst weniger Probleme, als dass sie neue schafft. Insbesondere in der zahnmedizinischen Versorgung von Alten, Behinderten und Pflegedürftigen wäre ein Unterlaufen der derzeitig geforderten Qualifikationen und der geltenden Standards kontraproduktiv.

\section{Mit kollegialen Grüßen}

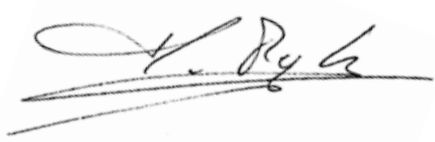

drs. Hub. van Rijt 